\title{
Twin-cutter form turning of commutator rings using cutters' progressive motion piezoelectric engines
}

\author{
Andrey Vozhzhov ${ }^{1, *}$, Eugene Pashkov $^{1}$, Vasiliy Golovin ${ }^{1}$, and Pavel Florya ${ }^{1}$ \\ ${ }^{1}$ FSAEO HO Sevastopol State University, 299053, 33 Universitetskaya str., Sevastopol, Russia
}

\begin{abstract}
The option of realization of the technological scheme of twin-cutter form turning via use of piezoelectric engines of progressive motion is hereby proposed. The cutting force vector constitutes the external perturbation effect. The scheme, design, transfer function of the cutter's piezoelectric engine of progressive motion is hereby presented.
\end{abstract}

Special attention is drawn to questions of improving the quality of the surface and the accuracy of particularly complex precision parts processing in the instrumentmaking industry. Various new materials, processing of which is associated with certain difficulties, constantly appear, the shape, surface accuracy and quality requirements to the part's surface become more complex, that's all demand the development of fundamentally new processing technologies, as the possibilities of traditional processing technologies in certain cases are limited and inefficient.

One of the most important elements that determine the reliability and durability of various control systems is the current-collecting unit - electrical contact point.

Due to the lack of reliable and durable contacts, it is often necessary to reduce the life time of expensive systems and products in general.

A special place among electrical contact points is taken by a low-current sliding contact points, that are widely used in the aircraft, space, ship control systems, in manufacturing automation equipment.

Low-current sliding contact points consist of a cylindrical commutator and brushes. Mechanically, a sufficiently large group of rings may be characterized as cylindrical with profile flute (Fig. 1). Manufacturing complexity of such parts is associated with both high requirements for accuracy and quality of the surface, and with their miniaturized dimensions

The miniaturization of low-current sliding contact points structures is associated with the economy of noble metals used for their production, reduction of mass and size of the contact point units. Another reason for the miniaturization of the structure is the search for optimal conditions for contacting and operating parameters that increase reliability and durability. However, when the size of contact points decreases, their manufacture becomes more expensive, defect ratio increases, objectiveness of evaluation with regard to reliability and life cycle of contact points is significantly reduced.

Flute form turning operation is performed on special lathes machines with improved precision. Concurrently twin-cutter form turning is one of the effective technological solutions. Flute treatment by two simultaneously working cutters reduces the negative influence of cutting force deformation to minimum, thus leads to accuracy increase and reduces the height of the chatter marks on the processing surface.

Processing based on opposite placement of two cutters is the simplest solution. This will allow the process of dividing the feed between the cutters to be performed automatically at any numerical values of the feed amount since one cutter will always lag behind the other by half the revolution in the direction of rotation. Herewith the high processing capacity is ensured as well as sharpening and adjustment of the cutters are greatly simplified.

In practice, this method is implemented in two fundamentally different ways. Practical implementation of the method assumes a sequential arrangement of two cutters one behind the other, while the second cutter may operate in parallel with the first or may enter into operation at the final stage of first cutter processing. If both tools are statically adjusted to the same processing dimension, increased elastic release in the section of the first cutter will result not only the removal by the second cutter of the processed surface roughness left by the first cutter, but it will also cut off some of the left (due to the first cutter release) processing allowance.

The implementation of high-precision form turning of miniature parts, requires the creation of high-precision tracking drives of progressive motion, operating in the micro- and nanometer range with an accuracy from tenths to hundredth of a micrometer. Rotation microengines with mech-electric converters of rotary motion to progressive motion do not provide the required high accuracy and speed.

\footnotetext{
${ }^{*}$ Corresponding author: 0506773532@mail.ru
} 


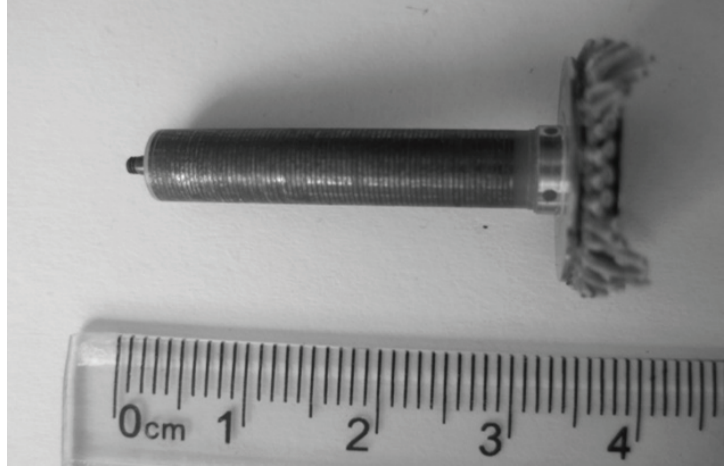

a)

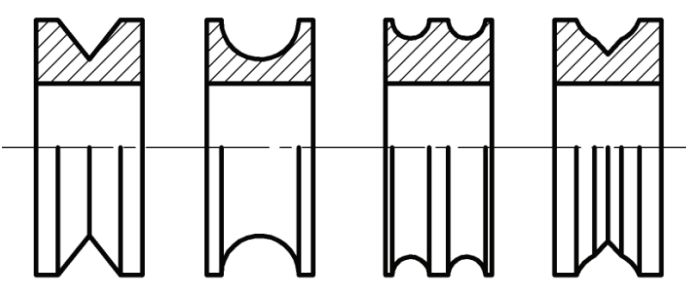

b)

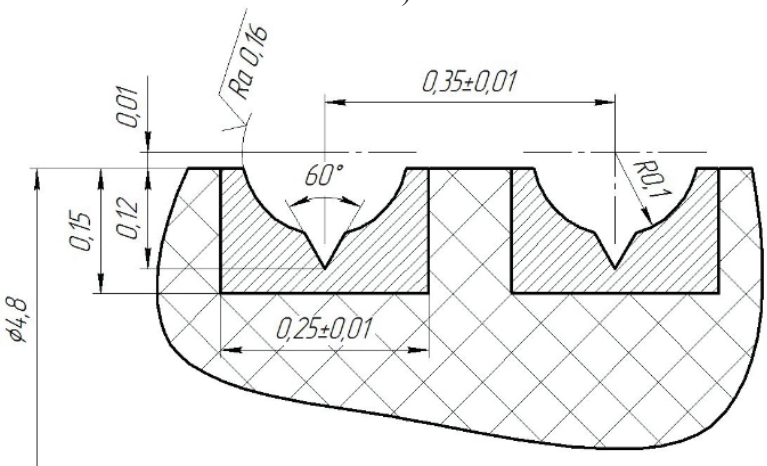

c)

Fig. 1. Collector (a), rings of low-current sliding contact points (b) and a typical flute profile for a brush (c).

The most effective for these purposes is the use of piezoelectric engines of progressive motion (PEPM), in which the conversion of electrical energy into mechanical energy is carried out due to the inverse piezoelectric effect [1].

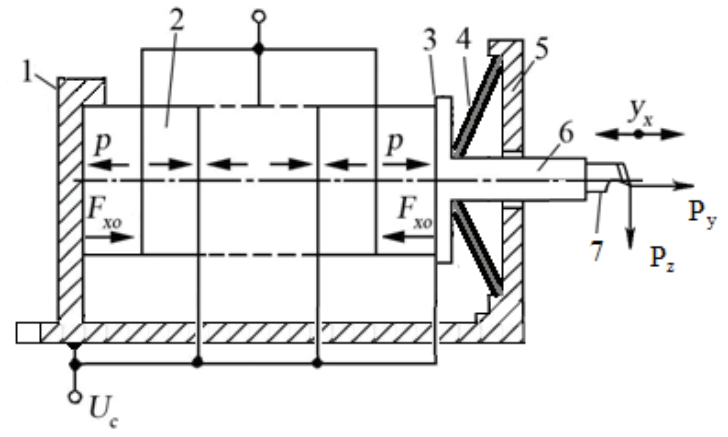

Fig. 2. Scheme of stack type PEPM for cutters

According to the constructive features, PEPMs are quite diverse. The simplest PEPMs, from the constructive aspect, are the tubular and stack constructions. Stacks are collected from individual piezoelements (PE) in the form of rods, disks, washers with a PE number in one stack, reaching 50 and over. The expediency of the PEPM stack construction is determined by the need to reduce the control voltage $U_{y}$, as $U_{y}=E_{d} \cdot a$, where $E_{d}$ is the allowed electric field intensity of the piezoceramics; $a$ PE thickness [1]. The allowed electric field intensity $E_{d}$ is limited at a certain level. For example, for a twocomponent piezoceramic (like PZT $\mathrm{Pb}\left(\mathrm{Zr}_{0,55} \mathrm{Ti}_{0,45}\right) \mathrm{O}_{3}$ ) $E_{d . s}=85 \mathrm{~V} / \mathrm{mm} ; \quad f=100 \mathrm{kHz}, \quad$ and for a multicomponent ceramics (like PKR) $E_{d . \mathrm{B}} \approx 60 \ldots 140 \mathrm{~V} / \mathrm{mm}, f=100 \mathrm{kHz}$. Therefore in order to reduce $U_{y}$ it is necessary to reduce the thickness of PE. At the same time, the stack is formed by gluing the unipolar surfaces of individual PE with the thicknesses $a \approx 1 \mathrm{~mm}$. A metal foil placed in the place of gluing is acting as an electrode. PE is connected mechanically in series, and electrically in parallel.

Fig. 2, [1] stipulates the scheme of stack type PEPM from disk shaped PE.

In order to increase the mechanical strength and stability PE engine have to be assembled by means of a prestressed elastic element 4 (for example, a rubber ring) installed between the flanges 3 and 5 , that are pressed against each other by the force $F_{x o}$ applied to the flange 1 . The output element of the PEPM the cutter 6 is kinematically connected to the operating device SP. The mechanical tension $T_{x o}$ in PE reaches $(1 \ldots 3) \cdot 10^{7} \mathrm{~N} / \mathrm{m}^{2}$; $\left|T_{x o}\right|=F_{x o} / A$; where $A$ is the cross-sectional area of the PE.

An increase of the number of PEs in the stack increases the power of the PEPM and the cumulative value of movement of the output element - the cutter.

The static characteristic (Fig. 3) of a stack-type PEPM in the quasistatic linear conditions, with $\pm U_{y}$ can be approximately described by the expression [1]

$$
\Delta y_{x} \approx k_{x} \cdot n \cdot d_{i j} U_{y},
$$

where $k_{x}$ - structural-technological ratio, taking into account the cycle and the operating mode of the engine; $n$ - the number of PE in the stack; $d_{i j}$ - piezoelectric unit; $i j$ - index marks, determined by the PE polarization, affecting the deformation direction of PE.

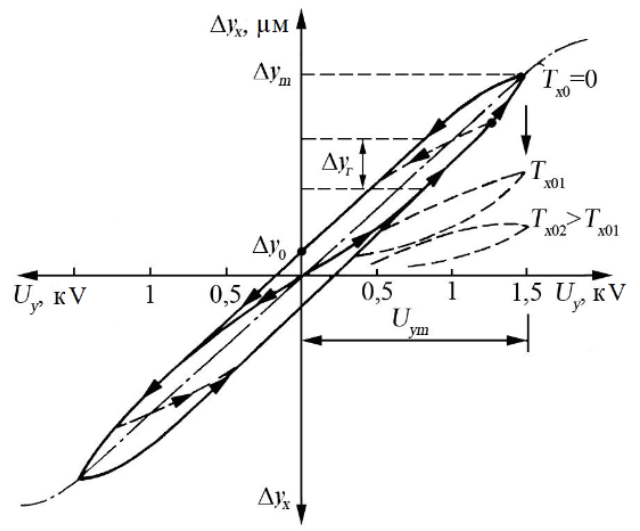

Fig. 3. Static characteristic of a stack-type PEPM 
Minimum piezoelectric unit for piezomaterials of the barium titanate system at a temperature of no higher than $85^{\circ} \mathrm{C}$ is $d_{31}=1,3 \cdot 10^{-8} \mathrm{~m} / \mathrm{V}$; for piezomaterials of the lead titanate system $-d_{31}=0,2 \ldots 0,3 \cdot 10^{-8} \mathrm{~m} / \mathrm{V}$ (at a temperature of $20^{\circ} \mathrm{C}$ ) and $d_{31}=0,4 \ldots 0,5 \cdot 10^{-8} \mathrm{~m} / \mathrm{V}$ (at a temperature of $40^{\circ} \mathrm{C}$ ) (the first index $i=3$ refers to an electric field directed parallelly to the thickness, the second index, $j=1$ refers to the deformation, perpendicular to the polarization direction) [5].

The sensitivity to the movement of the PEPM in quasistatic mode is determined by the ratio $\Delta y_{x} / U_{y}$, which is approximately $1,0 \mu \mathrm{m} / \mathrm{kV}$ for a single PE with $a=1 \mathrm{~mm}$ from modern piezomaterials (PZT) $\left(T_{x}=0\right)$ [2]. Variation limits $U_{y}$ in stack PEPM are $(1 \ldots 20) \cdot 10^{2} \mathrm{~V}$; the value $\Delta y_{x}$ at $U_{y \text { max }}$ reaches $50 \mu \mathrm{m}$ or more.

When the control voltage is varied in the range $-U_{y \text { max }} \leq U_{y} \leq+U_{y \text { max }}$, the static characteristic $\Delta y_{x}=f\left(U_{y}\right)$ is nonlinear and has the form of hysteresis loops (fig. 3). In this case values $k_{x}$ and $\Delta y_{x} / U_{y}$ are nonlinear variable parameters with maximum values in the initial (rectilinear) part of the characteristic, which may be approximately described by one of the known analytical expressions for hysteresis loops, for example, of the form (longitudinal polarization)

$$
\begin{aligned}
& \left.\Delta y_{3}\right|_{T_{3}=0} \approx k_{1} U_{y}+k_{2} U_{y}^{3}+k_{3} U_{y}{ }^{5}- \\
& -k_{4} U_{y m}\left(1-U_{y}{ }^{2} / U_{y m}{ }^{2}\right)^{n / 2} \operatorname{sign} \frac{d U_{y}}{d t},
\end{aligned}
$$

where $U_{y m}-$ is the amplitude value $U_{y} ; k_{1} \ldots k_{3}-$ approximation ratios, determined from the three selected points of the main experimental curve $\left(k_{1} \approx k_{x} n d_{33}\right.$; $\left.k_{2} \approx 0,75 \ldots 0,88 y_{m} ; \quad k_{3} \approx 0,95 \Delta y_{m}\right) ; \quad k_{4}=y_{0} / U_{y_{m}} ;$ $n=2,3,4,5 \ldots-$ value that depends on the shape of the experimental curve [3].

The transfer function of a PEPM as a harmonically linearized link will generally look:

$$
\begin{aligned}
& W_{\mathrm{H}}=\Delta y_{x 1} / U_{y}=q\left(U_{y_{m}}, \omega\right)+j q^{\prime}\left(U_{y_{m}}, \omega\right)= \\
& =A_{q}\left(U_{y_{m}}, \omega\right) \exp \left(j \varphi_{q} \times\left(U_{y_{m}} ; \omega\right)\right)
\end{aligned}
$$

where $A_{q}\left(U_{y_{m}}, \omega\right)$ is the transfer ratio; $\varphi\left(U_{y_{m}}, \omega\right)$ - phase shift characterizing the nonlinear delay; $q$ and $q^{\prime}$ are the ratios of harmonic linearization.

In the particular case of the control of a PEPM of a stack type with a constant voltage, its transfer function has the following approximate form [1]:

$$
\begin{aligned}
& W_{x}(p)=\frac{\Delta y_{x}(p)}{U_{y}(p)} \approx \frac{k}{T_{1}^{2} T_{2}^{2} p^{2}+T_{1}^{2} p^{2}+T_{2} p+1} \approx \\
& \approx \frac{k}{T_{0}^{2} p^{2}+2 \xi T_{0} p+1}
\end{aligned}
$$

where $k, T-$ transmission ratio and time constants, respectively; $\xi$-ratio of relative attenuation.

As it appears from expression (4), the PEPM is a thirdor second-order vibrational element, which is close to the conservative element for small values $\xi$.

In addition to the PEPM discussed above, other designs are known in which both the DC voltage control modes $U_{y}$ and the mechanical oscillation modes of the PE at a frequency $f_{p}$ are used. Mechanical vibrations of the $\mathrm{PE}$ are transformed into progressive motion of the moving part (cutter). If the frequency of oscillations of the PE $0<f_{p}<1 \mathrm{kHz}$, then the PEPM can be considered as a progressive mode one; when $1 \mathrm{kHz}<f_{p}<(1 \ldots 5) \mathrm{MHz}$ there is practically uniform parallel motion of the moving part (MP).

Therewith, traction forces up to $20 \mathrm{~N}$ are provided, which is sufficient for realization of the technological scheme of twin-cutter form turning [4].

PEPM provide high dynamic properties in step-bystep, start-stop modes. The range of step change in the progressive motion PEPM can be from $10^{-9}$ up to $10^{-6} \mathrm{~m}$ and more, the speed of movement $V_{c} \approx 0,1 \ldots 0,15 \mathrm{~m} / \mathrm{s}$.

The equation of motion has the following form (5)

$$
F_{T}-F_{\Sigma}=m \frac{d V}{d t}
$$

where $m$ - the effective dynamic mass of the entire moving system; $\frac{d V}{d t}-$ linear acceleration.

Frequency variations, amplitude or phase control methods can be used to change the speed of the PEPM. The development of a control model for the cutting process on the operation of twin-cutter form turning and the implementation of the control over the process are the goal of further research.

\section{References}

1. R.G. Dzhagupov, Piezoceramic elements in instrumentation and automation (1986)

2. E.V. Pashkov, V.A. Kramar, A.A. Kabanov, Tracking drives of industrial process equipment: teaching guide (2013)

3. E.P. Popov, I.P. Paltov, Approximate methods for investigating nonlinear automatic systems (1960)

4. A.A. Vozhzhov, S.V. Borozdin, Simulation of force interactions in the formation of flute surfaces of collector rings of low-current sliding contacts, Izvestiya of the Tula State University. Engineering Sciences, Issue 8. Part 1, p. 187-193 (2016)

5. I.A. Gluzman, Piezoceramics (1972). 